\title{
A PERFORMANCE COMPARISON OF THREE APPROACHES IN BATCH TEMPERATURE CONTROL
}

\author{
A. Shamekh and A. Altowati \\ Electrical and Electronic Engineering Department \\ University of Garyounis, Benghazi- Libya
}

\begin{abstract}
This article proposes the use of Output Error Model Predictive Control (OE-MPC) as a standard MPC technique for temperature control applications. Least squares of an output error criterion embedded with MPC was implemented in the basis of the control law design. The OE model was exploited to minimize the plant-model mismatch, and therefore the controller accuracy is significantly improved. The paper compares the performance of the suggested controller with the performance of MPC based ARX (Autoregressive with exogenous input) model, and Generic Model Control (GMC) in temperature control of an exothermic batch process simulation. The work identifies the features of the considered algorithms in a comparative approach.
\end{abstract}

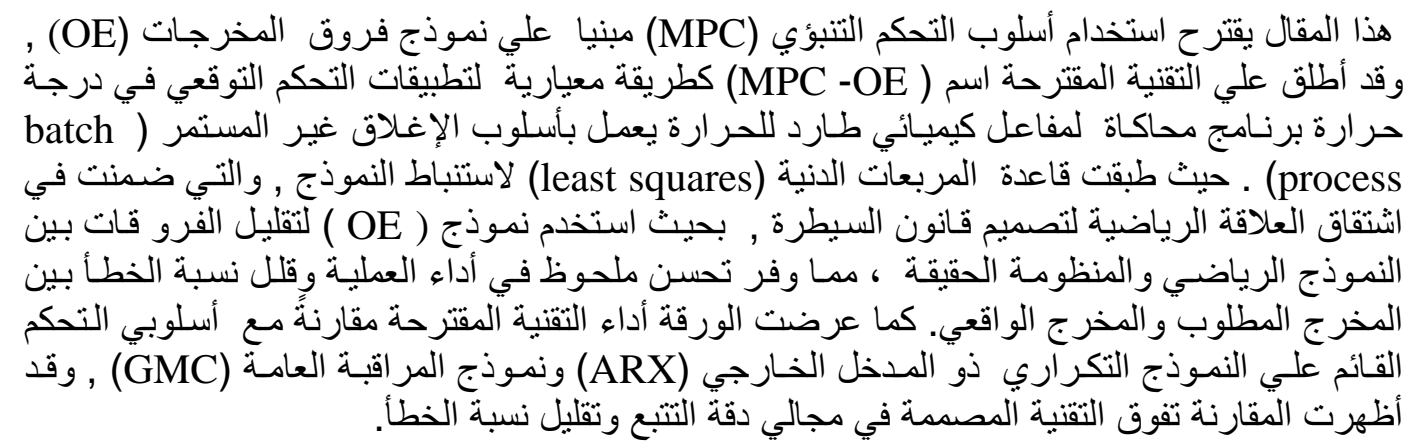

Keywords: Batch process, adaptive control, model predictive control, nonlinear control, system identification

\section{INTRODUCTION}

Batch control is an active area of research, and it has received much of concern in the literature. A batch process is one in which a sequence of operations are carried out over a period of time on a specific item or mass of material. It differs from a continuous process, in which all operations occur simultaneously and the material being processed is not divided into units. Batch processes are considered to be an economically effective approach to manufacturing as they are able to respond to varying product quantities. Consequently they are best suited for the manufacture of low volume, high value products such as speciality chemicals, polymers, pharmaceuticals, food, multiphase materials and blends. To maintain efficient batch production and consistent operation from shift to shift, it is necessary for the process parameters to be precisely supervised. Having multiple products with several stages from the same vessel may significantly complicate operations due to the varying dynamic nature of the batch processing. A strict control for reactor temperature is a key requirement in many batch manufacturing to ensure the production quality and to fulfil safety instructions. However, this is usually not easy task due to the fact that the batch processes are typically highly nonlinear processes with complex dynamic behavior.

Implementation of MPC methodology in batch process control was presented by several articles over the past two decades. These including Rafalimana et al. (1992), Rho et al. (1998), and Bindlish and Rawlings (2003). Flores-Cerrillo and MacGregor (2005) developed Latent Variable Model Predictive Control (LV-MPC) where imputation of missing data using of a dynamic Principle Component Analysis (PCA) model was utilized to determine the control moves. The authors argued that 
the proposed control law could provide a control action similar to the standard MPC. Golshan et al., (2010) mentioned that in LV-MPC, a PCA model can be built either in moving window style where the constructed model is applicable to every time point along the batch-wise unfolded dataset, or in a multiphase criterion in which the batch is divided into a number of phases and a specific PCA model should be identified for each particular phase. Thereby, during the batch control these models are employed within their respective phases. Shamekh and Lennox (2010) discussed the issue of LV-MPC stability and displayed that the controller performance cannot be consistent for all batches. The article attributes the controller instability to the loss of orthogonality of the PCA model loading matrix that resultant from the data decomposition.

In this paper, the effect of incorporating various models within the model based control framework was investigated and conclusion drawn. The design of a standard MPC coupled with OE model for temperature control of an exothermic batch process is presented. The work also implements ARX model included with MPC (ARX-MPC) and reviews GMC technique with on-line released heat estimate. A comparison has been made in the sense of minimizing a mean absolute error (MAE) statistic index for the used algorithms. The remaining of this work is organized as; section 2 discusses the OEMPC algorithm with ARX-MPC. In section 3, GMC is briefly reviewed, whereas section 4 presents design considerations and results with appropriate analysis. Ultimately, section 5 summaries the conclusions.

\section{OE-MPC DESIGN}

\subsection{OE Model structure and identification}

Linear MPC requires an explicit model of the process which is used to estimate the process outputs in specified time instants in the future (prediction horizon). These estimates are then used to determine appropriate control actions (MPC moves) in a predefined range (control horizon). The MPC moves are computed such that they will drive the controlled system to a certain mode of operation which is prespecified by the set points for the control variables (reference trajectories).

The issue of estimator bias is an appealing problem in control engineering. The identification of model parameters can be achieved using many techniques including Ordinary Least Squares (OLS), recursive least squares, or partial least squares. OLS is an unbiased technique, which assumes that a linear relationship describes the system variables. However, this does not mean that the unbiased regression coefficients have minimum variance errors (Frank, 1987). The output error method creates an unbiased model by assuming that an undisturbed output, $\hat{y}(t)$, can replace the output signal $y(t)$ during the identification stage. However, this is normally valid provided that input and output signals are not mutually correlated (Ljung, 1999). In this article the following OE model transfer function was utilized in the model structure (Zhu, 2001):

$G\left(q^{-1}\right)=\frac{B\left(q^{-1}\right)}{A\left(q^{-1}\right)}=\frac{b_{1} q^{-1}+\ldots . .+b_{n} q^{-n}}{1+a_{1} q^{-1}+\ldots . .+a_{n} q^{-n}}$

where

$\frac{y(t)}{u(t)}=G\left(q^{-1}\right)$

The output error is then defined by:

$$
\varepsilon_{o e}(t)=y(t)-\frac{B\left(q^{-1}\right)}{A\left(q^{-1}\right)} u(t)=y(t)-\hat{y}(t)
$$

To avoid the problem of input-output being correlated under feedback action, independent components of PRBS were added to the system input at the stage of model training. However, this should be performed such that the process constraints are not violated. Flores-Cerrillo and MacGregor (2005) used MAE index to assess the amplitude of the PRBS to ensure that is quite small so that operation of the batch was not significantly upset. This criterion was applied in this work, whereas PID control of settings (Aziz et al, 2000) $K_{c}=13.5381, \tau_{I}=28.75, \tau_{D}=0.406$ was employed to supervise the batch during the collection of the training data. The resultant model was then integrated with MPC algorithm to calculate the control profile that is required to drive the system to follow the desired trajectory. As mentioned before and to emphasize the improvement of using $\mathrm{OE}$ model, MPC embedded with an ARX structure is also investigated in this work. The ARX model of first order dynamic polynomial with forth order input polynomial was identified with one sampling interval delay based on the least squares principle. Generally, the ARX structure is given by (Ljung, 1999)

$$
y(t)=-\sum_{j=1}^{n} a_{j} y(t-j)+\sum_{j=1}^{n} b_{j} u(t-j)+e(t)
$$

which is equivalent to

$$
A\left(q^{-1}\right) y(t)=B\left(q^{-1}\right) u(t)+\varepsilon(t)
$$

Where $y(t)$ and $u(t)$ are the output and input signals respectively. $A$ and $B$ are polynomials in the backwards shift operator $\left(q^{-1}\right)$ as follows:

$$
\begin{aligned}
& A\left(q^{-1}\right)=1+a_{1} q^{-1}+\ldots . .+a_{n} q^{-n} \\
& B\left(q^{-1}\right)=b_{1} q^{-1}+\ldots . .+b_{n} q^{-n}
\end{aligned}
$$

$\varepsilon(t)$ is the residual or error signal between the actual observed output and the estimated one. 


\subsection{MPC design}

In control design, MPC basically identifies future control action by minimizing a specific objective function, which is normally quadratic. Minimizing the error between the predicted output and set point and minimizing the control action that should be taken to achieve the first task is the essence mechanism of any MPC technique. The following cost function, as illustrated by Clarke et al. (1987), was implemented in the control law design.

$J=\sum_{j=1}^{p} \alpha_{j}\left(y_{r}(k+j / k)-\hat{y}(k+j / k)\right)^{2}+\sum_{j=1}^{m} \beta_{j} \Delta u(k+j-1 / k)^{2}$

Where $J$ is the index of the objective function to be minimized, $k$ is the sampling instant, $p$ and $m$ are the prediction and control horizons, respectively. The desired trajectory and estimated future output values in Equation (5), are represented by $y_{r}$ and $\hat{y}$ respectively, while $\alpha_{j}$ and $\beta_{j}$ denote the weighting parameters of the controlled and manipulated variables respectively. Whereas $\Delta u$ represents the change in manipulated variable from a time instant to another. The objective function in this design is subjected to two types of constraints that are typically given as (Camacho, 2005):

Input constraints (actuator limitations), which describe saturation and minimum and maximum rate of change of the manipulated signals.

$$
\begin{array}{cc}
\Delta u_{\min } \leq \Delta u(k+j / k) \leq \Delta u_{\max } & 0 \leq j \leq m-1 \\
u_{\min } \leq u(k+j / k) \leq u_{\max } & 0 \leq j \leq m-1
\end{array}
$$

Output constraints (operation limitations) which usually address constraints on product qualities and other issues relating to safety.

$$
\begin{array}{lc}
\Delta y_{\min } \leq \Delta y(k+j / k) \leq \Delta y_{\max } & 0 \leq j \leq p \\
y_{\min } \leq y(k+j / k) \leq y_{\max } & 0 \leq j \leq p
\end{array}
$$

\section{GMC CONTROL}

Lee and Sullivan (1988) proposed GMC as a nonlinear control algorithm. The technique is based on the on-line heat released estimation method, which was presented by Jutan and Uppal (1984). Cott and Macchietto (1989) reported several advantages of using GMC in the temperature control for batch processes, which are as: 1) it is a modelbased control strategy, where the process model is used directly in the control law algorithm;2) linearization is not necessary; 3) the GMC algorithm uses the rate of change in controlled variable directly in the feedback control. It is therefore able to track the heat-up and cool-down periods effectively; 4) it provides feed forward action, which is explicitly integrated with the feedback control. The use of a neural network model to update the GMC was implemented by Aziz et al., (2000) in batch temperature control. Mujtaba et al., (2006) compared the performance of a dynamic neural network (NN) estimator coupled with GMC, direct inverse control (DIC), and internal model control (IMC) for controlling the temperature of an exothermic batch reactor. This work utilizes the same estimate technique that was applied by Cott and Macchietto (1989) to identify the realised heat. However, it implements a different profile trajectory.

A dynamic process model is used by the GMC method. The control law is obtained by solving the estimated process model of the derivative of the controlled variable $\left(T_{r}\right)$. By setting this estimate equal to proportional-integral action operating on the process error signal $\left(T_{r s p}-T_{r}\right)$. $T_{r s p}$ represents the desired set-point trajectory. The algorithm as introduced by Cott and Macchietto (1989), is as follows:

$\frac{d T_{r}}{d t}=K_{1}\left(T_{r s p}-T_{r}\right)+K_{2} \int_{0}^{t}\left(T_{r s p}-T_{r}\right) d t$

where $K_{1}$ and $K_{2}$ are the GMC's tuning parameters. By neglecting the amount of heat retained in the reactor walls, the energy balance around the reactor contents can be expressed as:

$\frac{d T_{r}}{d t}=\frac{Q+U A\left(T_{j}-T_{r}\right)}{W C p}$

where $W$ is the weight of the reactor contents, $C p$ is the mass heat capacity of the reactor contents, $U$ is the heat transfer coefficient, $A$ is the heat transfer area, $T_{j}$ is the jacket temperature (manipulated variable), and $Q$ is the heat released by the reaction. By substituting equation (6) into equation (7), this yields:

$$
T_{j}=T_{r}+\frac{W C p}{U A}\left(K_{1}\left(T_{r s p}-T_{r}\right)+K_{2} \int_{0}^{t}\left(T_{r s p}-T_{r}\right) d t\right)-\frac{Q}{U A}
$$

Equation (8) gives the desired manipulated variable trajectory for the reactor temperature to follow the desired set-point. In order to implement this algorithm in the digital form, the integral action should be approximated by an arithmetic summation process as:

$$
\int_{0}^{t}\left(T_{r s p}-T_{r}\right) d t=\sum_{0}^{k}\left(T_{r s p}-T_{r(k)}\right) \Delta t
$$


where $\Delta t$ is the sampling period, and $k$ is the sampling instant. From equation (9), equation (8) can be rewritten as:

$T_{j(k)}=T_{r(k)}+\frac{W C p}{U A}\left(K_{1}\left(T_{r s p}-T_{r(k)}\right)+K_{2} \sum_{0}^{k}\left(T_{r s p}-T_{r(k)}\right) \Delta t\right)-\frac{Q_{(k)}}{U A}$

Cott and Macchietto (1989), Arpornwichanop et al. (2005), Aziz et al., (2000), and Mujtaba et al., (2006) stated that equation (8) does not take into account the jacket dynamics, and this might cause sluggish control, therefore a first order model, which illustrated in equation (11) is used in order to obtain the jacket set point.

$T_{j s p(k)}=T_{j(k-1)}+\tau_{j}\left(\frac{T_{j(k)}-T_{j(k-1)}}{\Delta t}\right)$

It is important to mention that this procedure was also implemented in application of MPC applications.

Similarly to the work of Cott and Macchietto (1989), the deterministic on-line energy balance was utilized in this work to estimate $\frac{Q}{U A}$. Using this, the number of parameters to be calculated is minimized into a single term $\left(\frac{W C p}{U A}\right)$. The heat released is obtained from equation (7) as follows:

$\frac{Q}{U A}=\frac{W C p}{U A} \frac{d T_{r}}{d t}-\left(T_{j}-T_{r}\right)$

Where $\frac{d T_{r}}{d t}$ is estimated from the direct measurement of $T_{r}$. Cott and Macchietto (1989) stated that numerical differentiation is very sensitive to measurement errors and therefore it may have a detrimental effect on the performance of the estimator. They suggested using a high order difference equation for calculating the derivative, and then a low pass filter to cut-off the high frequency components. The authors applied a three term difference equation that was used by Jennings (1964) and exponential filters with time constants of $\tau_{f}=1 \mathrm{~min}$ in both the temperature measurements and the estimate of $\frac{Q}{U A}$. The filtering signals were applied for estimation purposes only, whereas the GMC control law uses the real observed values of $T_{r}$ and $T_{j}$. The estimator details (subscript $f$ indicates the filtered signals), as described by Cott and Macchietto (1989) are as follows:

$$
\begin{aligned}
& T_{r f(k)}=T_{r f(k-1)}+\frac{\Delta t\left(T_{r}-T_{r f(k-1)}\right)}{\tau_{f}} \\
& T_{j f(k)}=T_{j f(k-1)}+\frac{\Delta t\left(T_{j}-T_{j f(k-1)}\right)}{\tau_{f}} \\
& \frac{d T_{r f(k)}}{d t}=\frac{3 T_{r f(k)}-4 T_{r f(k-1)}+T_{r f(k-2)}}{2 \Delta t} \\
& \left(\frac{Q}{U A}\right)_{(k)}=\left(\frac{W C p}{U A}\right)\left(\frac{d T_{r f(k)}}{d t}\right)+T_{r f(k)}-T_{j f(k)} \\
& \left(\frac{Q}{U A}\right)_{f(k)}=\left(\frac{Q}{U A}\right)_{f(k-1)}+\frac{\Delta t\left(\left(\frac{Q}{U A}\right)_{f(k)}-\left(\frac{Q}{U A}\right)_{f(k-1)}\right)}{\tau_{f}}
\end{aligned}
$$

It is important to note that with this version of GMC there is no off-line training for model building as in the former techniques and instead the released heat estimate is performed on-line in coherently with control law calculation.

The GMC algorithm is normally tuned with consideration of the desired controlled variable profile. Lee and Sullivan (1988) proposed two parameters ( $\xi$ and $\tau$ ) for tuning $K_{1}$ and $K_{2}$, which were defined as follows:

$$
\begin{aligned}
& K_{1}=\frac{1}{\tau^{2}} \\
& K_{2}=\frac{2 \xi}{\tau}
\end{aligned}
$$

Cott and Macchietto (1989) specified $\xi=10$ and $\tau=80 \mathrm{~min}$, which was based on the chart provided by Lee and Sullivan (1988). In this study, however a different temperature set-point profile was used and for these conditions, an improved control was obtained with $\xi=3.5 \times 10^{-6}$ and $\tau=1 \mathrm{~min}$. This was achieved by conducting few experiments.

\section{DESIGN CONSIDERATIONS AND SIMULATION RESULTS}

\subsection{Design considerations}

This work uses simulation of the mechanistic description of an exothermic batch reactor that was described by Cott and Macchietto (1989) and then exercised by several authors including Aziz et al., (2000), Arpornwichanop et al. (2005), FloresCerrillo and MacGregor (2005), and Mujtaba et al., (2006). Briefly, in this process, two reactants A and $\mathrm{B}$ are mixed in a reactor to produce a product, $\mathrm{C}$. A secondary reaction produces $\mathrm{D}$, which is an 
undesired product. The reactions taking place in this process are described as follows:

$A+B \stackrel{k 1}{\longrightarrow} C$

and

$A+C \stackrel{k 2}{\longrightarrow} D$

$k_{1}$ and $k_{2}$ are the Arrhenius constants. The process model is listed in appendix A. In the simulation of all control techniques, 150 min was selected for the batch duration with 0.5 min sampling period. The reactor temperature was corrupted with normally distributed random noise with a standard deviation of $\sigma=0.2$ in the case MPC designs. In OE-MPC, a first order output error model for the dynamic and input polynomial was identified from three batch training. The reactor temperature $\left(T_{r}\right)$ and jacket temperature set point ( $T_{j s p}$ ) were used as the output and input signals respectively for the system identification. 40 and 15 were used for the prediction and control horizons respectively, non-weighted control moves were performed with 10 for the output weight. The upper $\left(120^{\circ} \mathrm{C}\right)$ and lower $\left(15^{\circ} \mathrm{C}\right)$ jacket temperature limits were considered as the system constraints. It should be noted that these design specification were also applied for the ARX-MPC design.

For persistence excitation purposes in model training data, as discussed before, PRBS was added to the PID output according to MAE statistic. In the excitation assessment, the MAE index was calculated firstly for the reactor temperature controlled by PID without PRBS excitation, and then compared with others, which were subjected to the PRBS excitation. The MAE is given by (Flores-Cerrillo and MacGregor, 2005):

MAE $=\sum_{i=1}^{N} \frac{\left|y_{c v, i}-y_{s p, i}\right|}{N}$

$y_{c v, i}$ denotes the controlled variable whereas $y_{s p, i}$ signifies set- point signal, and $N$ denotes the number of samples. In this application, $\mathrm{MAE}=34 \%$ was utilized.

\subsection{Simulation results and remarks}

Figures (1) and (2) show the performance of the designed MPC based OE and ARX models for 10 batches respectively. Obviously from the figures, OE-MPC does its task better than ARX-MPC. In quantitative sense, Table (1) compares MAE indices of OE-MPC and ARX-MPC. It demonstrates the suitability of using OE (unbiased) structure (the less MAE index value means the control loop has a better performance) over ARX (biased) one in control design problems. This is because unbiased models are usually capture the actual system behavior and hence provide a significant improvement in modeling accuracy over the biased models, which are typically highly inaccurate.

Figures (3) to (5) display the results of the GMC design. Figure (3) explains that GMC with on-line heat release estimate can provide acceptable results in the presence of very little noise. However, it is evident, from the MAE indices, that as the disturbance level increased the GMC accuracy is severely affected as shown in Figure (4) and (5). This could be regard as a weak point in this identification approach (numerical differentiation), although it is on- line technique and does not need dither signals and off-line training.

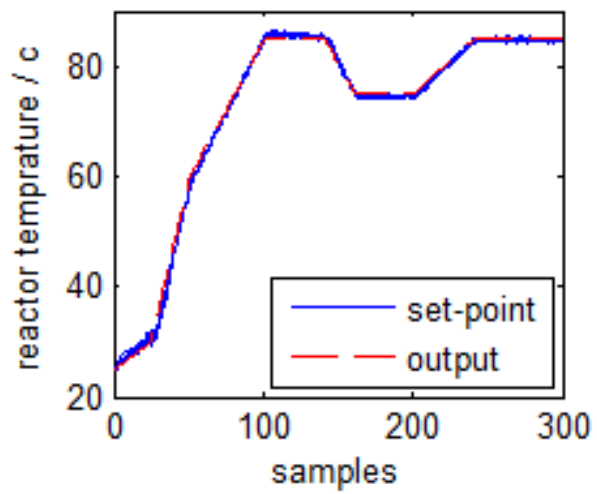

Fig.1 OE-MPC reactor temperature (10 batches)

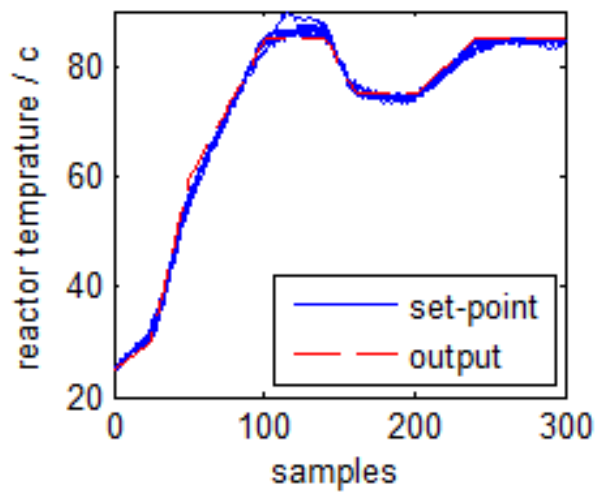

Fig.2 ARX-MPC reactor temperature (10 batches)

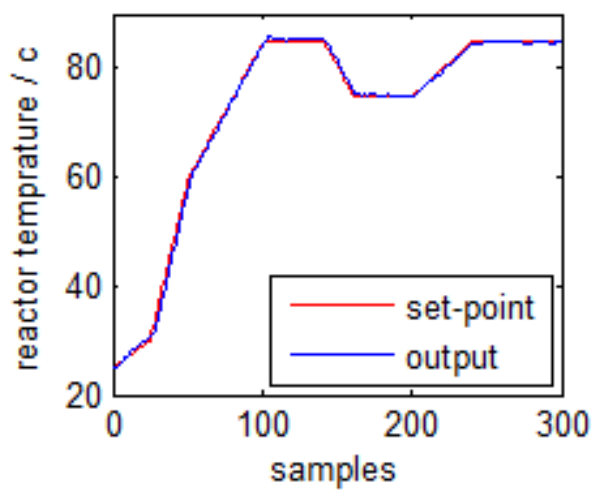

Fig.3 GMC reactor temperature $(\mathrm{MAE}=0.48$ at $\mathrm{Std}=0.01)$ 


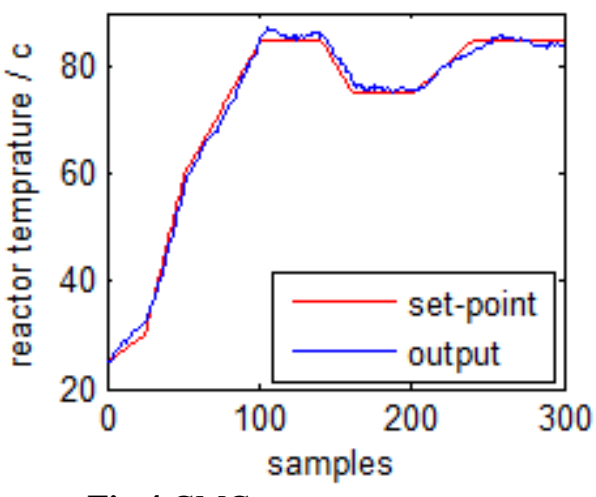

Fig.4 GMC reactor temperature (MAE=1.13at Std=0.15)

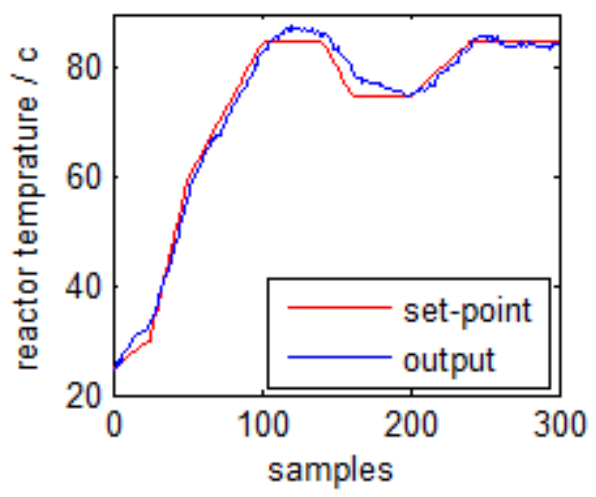

Fig.5 GMC reactor temperature (MAE=1.61 at $\mathrm{Std}=0.2)$

Table 1, MAE indices for OE-MPC and ARX-MPC

\begin{tabular}{|c|c|c|}
\hline Batch No. & $\begin{array}{c}\text { MAE } \\
\text { OE-MPC }\end{array}$ & $\begin{array}{c}\text { MAE } \\
\text { ARX-MPC }\end{array}$ \\
\hline Batch 1 & 0.5968 & 0.7060 \\
\hline Batch 2 & 0.5630 & 0.9232 \\
\hline Batch 3 & 0.6724 & 1.4526 \\
\hline Batch 4 & 0.5735 & 0.6934 \\
\hline Batch 5 & 0.6263 & 0.6386 \\
\hline Batch 6 & 0.5541 & 1.1557 \\
\hline Batch 7 & 0.6446 & 1.0050 \\
\hline Batch 8 & 0.6177 & 1.0225 \\
\hline Batch 9 & 0.5913 & 0.6859 \\
\hline Batch 10 & 0.5915 & 1.0887 \\
\hline
\end{tabular}

\section{CONCLUSIONS}

OE-MPC was introduced as an effective control technique that can provide a robust approach to temperature control in batch processes The control law synthesis and model construction procedure for OE-MPC were illustrated in a systematic and straightforward manner. Using the least squares output error method as the underlying model structure showed an adequate degree of robustness and reliability compared to ARX-MPC and GMC. The obtained results have indicated the feasibility of OE-MPC control which encourages implementation of this approach.

\section{REFERENCES}

[1] Arpornwichanop, A., Kittisupakorn, P., \& Mujtaba, I. M. (2005). On-line dynamic optimization and control strategy for improving the performance of batch reactors. Chemical Engineering and Processing, 44(1), 101-114.

[2] Aziz, N., Hussain, M. A., \& Mujtaba, I. M. (2000). Performance of different types of controllers in tracking optimal temperature profiles in batch reactors. Computers \& Chemical Engineering, 24(2-7), 1069-1075.

[3] Bindlish, R., \& Rawlings, J., B. . (2003). Target linearization and model predictive control of polymerization processes. AIChE, 49(11), 2885-2899.

[4] Camacho, E. F., \& Bordons, C. (2004). Model predictive control. London: Springer.

[5] Clark, D. W., Mohtadi, C. and Tuffs, P. S. (1987) Generalized Predictive Control-Part I. The basic algorithm, Automatica, 23 (2), pp.137-148.

[6] Cott, B. J. and Macchietto S. (1989) Temperature control of exothermic batch reactors using generic model control. Industrial Engineering chemistry research, (28), pp.11771184.

[7] Flores-Cerrillo, J., \& MacGregor, J. F. (2005). Latent variable MPC for trajectory tracking in batch processes. Journal of Process Control, 15(6), 651-663.

[8] Frank, I. E. (1987). Intermediate least squares regression method. Chemometrics and Intelligent Laboratory Systems, 1(3), 233-242.

[9] Jennings, W. (1964). First Course in Numerical Methods; The Macmillian Company. New York.

[10] Jutan, A. and Uppal A. (1984) Combined feed forward-feedback servo control scheme for an exothermic batch reactor, Industrial Engineering chemistry process design and development, (23), pp. 597-602.

[11] Golshan, M., MacGregor, J. F., Bruwer, M. J., Mhaskar, P. (2010). Latent Variable Model Predictive Control (LV-MPC) for trajectory tracking in batch processes, Journal of Process Control, 20, 538-550.

[12] Lee, P. L. and Sullivan G. R. (1988) Generic Model Control (GMC), Computers and chemical engineering, (12), No. 6, pp.573-580.

[13] Ljung, L. (1999). System identification : theory for the user. Upper Saddle River: Prentice Hall PTR.

[14] Mujtaba, I. M., Aziz, N., \& Hussain, M. A. (2006). Neural Network Based Modelling and Control in Batch Reactor. Chemical Engineering Research and Design, 84(8), 635644.

[15] Rafalimanana, A., Cabassud, M., Le Lann, M. V., \& Casamatta, G. (1992). Adaptive control 
A. Shamekh and A. Altowati "A Performance Comparison of Three Approaches in........."

of a multipurpose and flexible semi-batch pilot plant reactor. Computers \& Chemical Engineering, 16(9), 837-848.

[16] Rho, H.-J., Huh, Y.-J., \& Rhee, H.-K. (1998). Application of adaptive model-predictive control to a batch MMA polymerization reactor. Chemical Engineering Science, 53(21), 37293739.

[17] Shamekh A. and Lennox B.(2010). Latent variable MPC and its consistency in temperature control of batch processes., The 9th International Symposium on Dynamics and Control of Process Systems (DYCOPS 2010), IFAC. July 5-7, 2010 Leuven, Belgium.

[18] Zhu, Y. (2001). Multivariable system identification for process control. Amsterdam ; London: Pergamon.

\section{APPENDIX A}

The full dynamic model of the process is defined as follows:

$$
\begin{aligned}
& \frac{d M_{A}}{d t}=-k_{1} M_{A} M_{B}-k_{2} M_{A} M_{C} \\
& \frac{d M_{D}}{d t}=-k_{2} M_{A} M_{C} \\
& \frac{d T_{r}}{d t}=\frac{Q_{r}+Q_{j}}{M_{r}+C p_{r}} \\
& \frac{d T_{j}}{d t}=\frac{F_{j} \rho_{j} C p_{j}\left(T_{j s p}-T_{j}\right)-Q_{j}}{V_{j} \rho_{j} C p_{j}}
\end{aligned}
$$

Where

$W=M W_{A} M_{A}+M W_{B} M_{B}+M W_{C} M_{C}+M W_{D} M_{D}$

$$
M_{r}=M_{A}+M_{B}+M_{C}+M_{D}
$$$$
C p_{r}=\frac{C p_{A} M_{A}+C p_{B} M_{B}+C p_{C} M_{C}+C p_{D} M_{D}}{M_{r}}
$$$$
Q_{r}=-\Delta H_{1}\left(k_{1} M_{A} M_{B}\right)-\Delta H_{2}\left(k_{2} M_{A} M_{C}\right)
$$

$Q_{j}=U A\left(T_{j}-T_{r}\right)$

\begin{tabular}{|c|c|}
\hline Parameter & value \\
\hline & $\rho_{j}=1000$ \\
\hline $\mathrm{kJ} /\left(\mathrm{kmol}{ }^{\circ} \mathrm{C}\right)$ & $\mathrm{kg} / \mathrm{m}^{3}$ \\
\hline$C p_{B}=167.36$ & $\rho=1000$ \\
\hline $\mathrm{kJ} /\left(\mathrm{kmol}{ }^{\circ} \mathrm{C}\right)$ & $\mathrm{kg} / \mathrm{m}^{3}$ \\
\hline 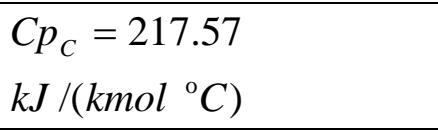 & $k_{1}^{1}=20.9057$ \\
\hline 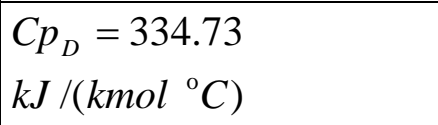 & $k_{1}^{2}=1000$ \\
\hline$M W_{A}=30 \mathrm{~kg} / \mathrm{kmol}$ & $k_{2}^{1}=38.9057$ \\
\hline$M W_{B}=100 \mathrm{~kg} / \mathrm{kmol}$ & $k_{2}^{2}=17000$ \\
\hline$M W_{C}=130 \mathrm{~kg} / \mathrm{kmol}$ & $V_{j}=0.6921 \mathrm{~m}^{3}$ \\
\hline$M W_{D}=160 \mathrm{~kg} / \mathrm{kmol}$ & $A=6.42 \mathrm{~m}^{2}$ \\
\hline$\Delta H_{1}=-41840 \mathrm{~kJ} / \mathrm{kmol}$ & $\tau_{j}=5$ \\
\hline$\Delta H_{2}=-25105 \mathrm{~kJ} / \mathrm{kmol}$ & $\Delta t=0.5 \mathrm{~min}$ \\
\hline $\begin{array}{l}C p_{j}=1.8828 \\
k J /\left(k g{ }^{\circ} \mathrm{C}\right)\end{array}$ & $r=0.5 \mathrm{~m}$ \\
\hline $\begin{array}{l}U=40.842 \\
k J /\left(\min m^{2}{ }^{\circ} C\right)\end{array}$ & $\begin{array}{l}F_{j}=0.348 \\
m^{3} / \min \end{array}$ \\
\hline
\end{tabular}

$$
A=\frac{2 W}{\rho r}
$$

The Arrhenius terms are given by

$$
\begin{aligned}
& k_{1}=\exp \left(k_{1}^{1}-\frac{k_{1}^{2}}{T_{r}+273.15}\right) \\
& k_{2}=\exp \left(k_{2}^{1}-\frac{k_{2}^{2}}{T_{r}+273.15}\right)
\end{aligned}
$$

Table (A.1), the model parameters 